\title{
MicroRNA-133b Ameliorates Allergic Inflammation and Symptom in Murine Model of Allergic Rhinitis by Targeting NIrp3
}

\author{
Lifeng Xiao Li Jiang Qi Hu Yuru Li \\ Department of Otolaryngology Head and Neck surgery, the First Affiliated Hospital of Harbin Medical \\ University, Harbin, China
}

\section{Key Words}

Allergic rhinitis $・$ Inflammation $\cdot$ microRNA-133b • Nlrp3 inflammasome

\begin{abstract}
Background: Emerging evidences indicate that post-transcriptional regulation by microRNAs is critical in allergic rhinitis (AR) pathogenesis. MircroRNA-133b (miR-133b) was recently suggested as a potential predictor of AR. However, the in vivo effect of miR-133b on AR is unclear. Methods: AR model was established in BALB/C mice by intraperitoneal sensitization and intranasal challenge with ovalbumin (OVA). MiR-133b agomir was then intranasally administrated to mice after OVA challenge for another 7 days. The symptom of nasal rubbing and sneezing were recorded after the last OVA challenge. Nasal mucosa tissues and serum were collected. MiR-133b expression, serum OVA-specific immunoglobulin E (IgE) concentration, proinflammatory cytokines (TNF- $\alpha$, IL-4, IL-5, IL-10 and IFN- $\gamma$ ) levels, and NIrp3 inflammasome activation were measured by RT-PCR, ELISA, western blotting or immunohistochemistry, respectively. Histopathologic changes were evaluated using hematoxylin and eosin and Sirius red staining. The luciferase activity and protein expression of Nlrp3 were also determined. Results: MiR-133b expression was significantly decreased in nasal mucosa of AR mice, which was restored by nasal administration with miR-133b agomir. Upregulation of miR-133b markedly reduced the concentration of OVA-specific IgE, the frequencies of nasal rubbing and sneezing, and the levels of cytokines (TNF- $\alpha$, IL-4, IL-5 and IFN- $\gamma$ ). Levels of IL-4, IL-5, IL-10 and IFN- $\gamma$ produced by cervical lymph node cells were significantly lowered in miR-133b agomirtreated mice. Moreover, miR-133b also appeared to strongly attenuate pathological alterations and eosinophils and mast cells infiltration in nasal mucosa. Notably, we demonstrated for the first time that miR-133b negatively regulated Nlrp3 expression through binding with the $3^{\prime}$ untranslated region of NIrp3. Consequently, infection of miR-133b in nasal mucosa remarkably suppressed the Nlrp3 inflammasome activation, as evidenced by reduced NIrp3, Caspase-1, ASC, IL-18 and IL-1 expressions. Conclusion: MiR-133b alleviates allergic symptom in AR mice by inhibition of Nlrp3 inflammasome-meditated inflammation. These findings provide us an insight into the potential role of miR-133b in relation to AR treatment.




\section{Cellular Physiology Cell Physiol Biochem 2017;42:901-912 \begin{tabular}{l|l} 
DOI: 10.1159/000478645 & and Biochemistry 2017 The Author(s). Published by S. Karger AG, Basel \\
Published onlne: June 26, 2017 & \begin{tabular}{l} 
www.karger.com/cpb \\
\cline { 2 - 3 }
\end{tabular}
\end{tabular} Xiao et al.: Inhibitory Effects of miR-133b on Allergic Inflammation}

\section{Introduction}

Allergic rhinitis (AR) is the most common noninfectious rhinitis driven by immunoglobulin E (IgE)-mediated immunological responses [1]. Many AR patients are often accompanied by comorbidities like chronic rhinosinusitis and asthma [2]. It not only largely affects social life, sleep and work, but also increases family and socioeconomic burden [1]. Despite rapid progress in the treatment of AR, such as immunotherapy, intranasal steroids, and antihistamines, $20 \%$ of AR patients already do not show sufficient subjective and objective improvement [3]. Accordingly, it is of foremost importance to develop potential pharmacological agents for the treatment of AR.

Emerging evidences have shown that inflammatory mechanisms play a key role in the development of AR [4]. The inflammatory response is associated with increased number of eosinophils, and upregulated levels of proinflammatory cytokines, such as TNF- $\alpha$, IL-4, IL-5 and IFN- $\gamma$ [5-7]. The interaction between these cytokines and glandular structures results in a variety of symptoms, including rhinorrhea, nasal obstruction, post nasal drainage, sneezing, and nasal irritation [8]. Interestingly, more recent studies have focused the role of innate immune-sensing receptors in inflammation diseases. The inflammasome is a cytoplasmic multiprotein that contains the nod-like receptor protein (Nlrp) family, adaptor protein ASC [9]. It triggers Caspase-1, IL-18 and IL-1 $\beta$ activation to execute inflammatory responses $[9,10]$. Nlrp3 inflammasome activation has been detected in nasal mucosa of AR rats [11]. Moreover, previous studies have demonstrated that caspase- 1 and IL-1 $\beta$, two key markers of Nlrp3 inflammasome, are also critical in the pathogenesis of AR [12,13]. These findings together suggest that Nlrp3 inflammasome may play an important role in regulating allergic inflammation of AR.

Allergic inflammation involves complicated molecular mechanism. Mounting investigations implicate that post-transcriptional regulation by microRNAs (miRs) is critical in AR pathogenesis [14-16]. Specifically, accumulating evidences suggest that miR-133b may be a novel potential predictor for inflammation. A previous study indicated that the changes of miR-133b expression might involve in the regulation of neuropathic pain, which were correlated with the expression of inflammatory cytokines [17]. Downregulation of miR-133b could be observed in the muscle of patients with inflammatory myopathy [18]. Moreover, a recent computational investigation using microarray analysis revealed that miR$133 \mathrm{~b}$ expression was reduced in the blood of AR patients, suggesting circulating miR-133b may be a biomarker in patients with AR [19]. However, to our knowledge there has been no other study addressing the effect of miR-133b on AR in vivo, except for a report describing the expression pattern of miR-133 in AR patients [19]. In this study, therefore, we examined the effects of miR-133b on allergic symptoms and inflammation in AR mice. Furthermore, our findings demonstrate that miR-133b determines Nlrp3 expression and regulates Nlrp3 inflammasome-mediated inflammation.

\section{Methods and Materials}

\section{Animals}

Eighty 8-week-old BALB/c mice were purchased from the Experimental Animal Center of Harbin Medical University and housed in a specific-pathogen-free biohazard containment facility maintained at 12 $\mathrm{h}$ dark/light cycle and $20 \pm 1^{\circ} \mathrm{C}$ room temperature with free access to food and water. The mice were divided into four groups: control, AR (AR mice treated with saline), negative (AR mice treated with mismatched agomir) and miR-133b agomir (AR mice treated with miR-133b agomir). This study was approved by the Committee on the Use and Care of Animals of Harbin Medical University.

AR model production

AR model was induced according to previously reported protocol shown in Fig. S1 [20]. Briefly, mice were sensitized by intraperitoneal injection of $200 \mu \mathrm{L}$ of saline containing $25 \mu \mathrm{g}$ ovalbumin (OVA) and 2 


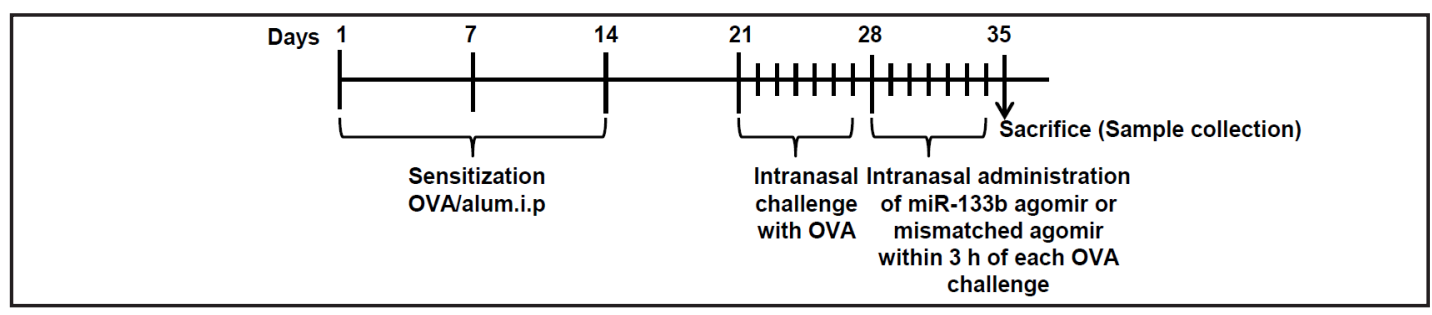

Fig. 1. Animal experimental design. BALB/c mice were sensitized by intraperitoneal injection of ovalbumin (OVA) and aluminum hydroxide (alum) on days 0,7 , and 14 to promote primary sensitization. One week after the last intraperitoneal injection, mice were intranasally challenged with OVA daily for a week for secondary immunization. MiR-133b agomir or mismatched agomir was intranasally administrated to mice before $3 \mathrm{~h}$ of OVA challenge on days 28-34.

mg of aluminum hydroxide (Sigma-Aldrich, St. Louis, MO, USA) on days 0,7 , and 14 to promote primary sensitization. One week after the last intraperitoneal injection, mice were intranasally challenged with 3\% OVA diluted in $20 \mu \mathrm{L}$ of saline daily for a week for secondary immunization. The control (normal) group was given saline containing no OVA and aluminum.

Intranasal administration of miR-133b agomir

MiR-133b agomir and mismatched agomir (MA) were constructed at the GeneChem Company (Shanghai, China). As displayed in Fig. 1, $5 \mathrm{pmol} / \mu \mathrm{L}$ miR$133 \mathrm{~b}$ agomir diluted in $20 \mu \mathrm{L}$ saline was intranasally administrated to mice before $3 \mathrm{~h}$ of OVA challenge on days 2834. The negative group was intranasally administrated with $5 \mathrm{pmol} / \mu \mathrm{L}$ MA diluted in $20 \mu \mathrm{L}$ saline, while the AR group was treated with saline.

Table 1. The primer sequences used for quantitative PCR

\begin{tabular}{lll}
\hline Genes & & Primers sequences (5' to $3^{\prime}$ ) \\
\hline \multirow{2}{*}{ MiR-133b } & Forward & CGGAACCAAGTCCGTCTTCC \\
& Reverse & ATATTGAGCTTTGCCAGCCC \\
& Forward & GGTGCCTATGTCTCAGCCTC \\
& Reverse & ATAGCAAATCGGCTGACGGT \\
IL-4 & Forward & CAAACGTCCTCACAGCAACG \\
& Reverse & AGGCATCGAAAAGCCCGAAA \\
IL-5 & Forward & AAATTCCTGTAGCGCAGGCT \\
& Reverse & ACCCTGATGCAACGAAGAGG \\
IFN- $\gamma$ & Forward & AGCCTCAGGAAGCGGAAAAG \\
& Reverse & CTCATTGAATGCTTGGCGCT \\
NIrp3 & Forward & GACCATCGGCCGGACTAAAA \\
& Reverse & CTTGCACACTGGTGGGTTTG \\
Caspase-1 1 & Forward & ACTGCTATGGACAAGGCACG \\
& Reverse & GCAAGACGTGTACGAGTGGT \\
ASC & Forward & GAAGCTGCTGACAGTGCAAC \\
& Reverse & AGGAGGAACAGTTAAGCGCC \\
IL-18 & Forward & ACGTGTTCCAGGACACAACA \\
& Reverse & GGCGCATGTGTGCTAATCAT \\
IL-1 $\beta$ & Forward & GTCAACGTGTGGGGGATGAA \\
& Reverse & AAGCAATGTGCTGGTGCTTC \\
GAPDH & Forward & GGTTGTCTCCTGCGACTTCA \\
& Reverse & CCCTAGGCCCCTCCTGTTAT \\
\hline
\end{tabular}

Quantitative real-time PCR

After euthanization, the nasal mucosa tissues of the mice were taken and frozen using liquid nitrogen. Total RNA was isolated using Trizol reagent (Thermo Fisher Scientific, Yokohama, Japan) according to the manufacturer's instructions. 2 ug of RNA was reverse transcribed using a RevertAid First Strand cDNA Synthesis Kit (Thermo Fisher Scientific). Quantitative real-time PCR was performed by miRcute miRNA qPCR kit (TIANGEN, Beijing, China) with a LightCycler 480 qPCR System (Roche, Basel, Switzerland). The specific target gene primers are listed in Table 1 . The reaction conditions were as follows: denaturation at $95^{\circ} \mathrm{C}$ for $10 \mathrm{~min}$, followed by 40 amplification cycles of $95^{\circ} \mathrm{C}$ for $10 \mathrm{~s}$ and $60^{\circ} \mathrm{C}$ for $1 \mathrm{~min}$. Target mRNA level was normalized by GAPDH, and the data was calculated using the $2^{-\Delta \Delta \mathrm{CT}}$ method.

\section{Symptom score}

30 min after the final OVA challenge on day 27, AR mice were received behavioural test as previous described before intranasal administration of miRNA agomir [4, 10]. The frequencies of rubbing and sneezing were recorded, and scores were calculated as follows: 1, wipes the nose slight for several times or sneezes less than 3 times; 2, repeatedly wipes the nose or sneezes more than 3 times and less than 10 times; 3, keeps rubbing from nose to face or sneezes more than 11 times. Animals not able to achieve to 


\section{Cellular Physiology Cell Physiol Biochem 2017;42:901-912 \begin{tabular}{l|l} 
and Biochemistry Published onlıne: June 26, 2017 & $\begin{array}{l}\text { C) } 2017 \text { The Author(s). Published by S. Karger AG, Basel } \\
\text { www.karger.com/cpb }\end{array}$ \\
\hline
\end{tabular}}

Xiao et al.: Inhibitory Effects of miR-133b on Allergic Inflammation

more than 4 scores were excluded from further study. Behavioural test was also performed within 30 min of OVA challenge on day 34.

Serum concentration of OVA-specific immunoglobulin E (IgE)

Mouse serum concentration of OVA-specific IgE were measured as previously described [4]. Briefly, blood samples were collected from the orbital venous plexus of anesthetized mice at the time of death. The serums were harvested from blood samples by centrifugation for $10 \mathrm{~min}$ at $1000 \times \mathrm{rpm}$ and stored at $-80^{\circ} \mathrm{C}$ until use. Serum samples at a dilution of $1 / 5$ with phosphate-buffered saline (PBS) were added to 96-well plates coated with purified anti-mouse IgE mAb (BD Pharmingen, San Jose, CA, USA), and incubated at $37^{\circ} \mathrm{C}$ for $2 \mathrm{~h}$. After washing with wash buffer, horseradish peroxidase (HRP)-labeled OVA was added to each well and incubated for $1 \mathrm{~h}$ at $37^{\circ} \mathrm{C}$. The plates were then incubated with 3,3',5,5'-tetramethylbenzidine (Moss Inc., Belfast, ME, USA) for $1 \mathrm{~h}$, and the reactions were terminated by adding $\mathrm{H}_{2} \mathrm{SO}_{4}$. Optical density (OD) was measured by a luminometer (AutoLumat Plus, Berthold Technology, Calmbacher, Germany) at $450 \mathrm{~nm}$. The concentration of OVA-specific IgE was determined by interpolation from a standard curve.

Lymph node cell restimulation with OVA in vitro

Cervical lymph node cell suspensions $\left(1 \times 10^{6} / \mathrm{ml}\right)$ in RPMI-1640 supplemented with $5 \%$ fetal calf serum (FCS), gentamycin and $\beta$-mercaptoethanol (all from Invitrogen, Carlsbad, CA, USA) were restimulated with OVA $(10 \mu \mathrm{g} / \mathrm{mL})$ for 4 days. Supernatants of the lymph node cell suspensions were collected for ELISA quantification.

Quantitative measurement of cytokines

To evaluate the allergic reactions, cytokines (TNF- $\alpha$, IL-4, IL-5, IL-10 and IFN- $\gamma$ ) were assayed in serum using ELISA kits (Abcam, Cambridge, MA, USA). Procedures were performed according to the manufacturer's instructions.

\section{Histological analyses}

Mice were euthanized under ketamine $(50 \mathrm{mg} / \mathrm{kg})$ and xylazine $(5 \mathrm{mg} / \mathrm{kg}$ ) anesthesia $24 \mathrm{~h}$ after the final intranasal sensitization. The heads of the mice were removal and immediately fixed in $4 \%$ paraformaldehyde for $24 \mathrm{~h}$ at $4^{\circ} \mathrm{C}$. The tissues samples were dehydrated in a series of increasing concentrations of ethanol and embedded in paraffin. $4 \mu \mathrm{m}$-thick sections of nasal mucosa tissues were stained with hematoxylin and eosin (H\&E) for histopathological examination. Sirius red staining was adapted to visual eosinophils infiltration. The number of eosinophils in the nasal mucosa was counted in six randomly selected fields at $200 \times$ magnification. For immunohistochemistry, after de-waxing and dehydration, sections were incubated with antibodies against Nlrp3, Caspase-1, ASC, IL-18 (1:50 dilution, Santa Cruz, Paso Robles, CA, USA) and IL-1 $\beta$ (1:100 dilution, Cell Signaling Technology, Beverly, MA, USA) overnight at $4^{\circ} \mathrm{C}$, followed by incubation with appropriate biotinylated secondary antibodies for $1 \mathrm{~h}$ at room temperature and 3,3' diaminobenzidine tetrachloride (DAB) substrate color development. Then the sections were counter-stained with hematoxylin and examined under a light microscope (BX51, Olympus, Tokyo, Japan) at 400×magnifications.

\section{Confocal microscopy}

To test the influx of mast cells into nasal mucosa, sections containing fixed tissues were incubated with a monoclonal rabbit anti-mouse tryptase antibody (1:100, Abcam, Cambridge, MA, USA), a known marker of mature mast cells, at $4^{\circ} \mathrm{C}$ overnight. After washing three times with PBS, the sections were then labeled with a FITC-conjugated goat anti-rabbit secondary antibody (1:200, Abcam) for $1 \mathrm{~h}$ at room temperature in darkness. All sections were stained with nuclear dye DAPI (Sigma) for $5 \mathrm{~min}$ at room temperature then observed under a laser scanning confocal microscopy (Leica TCS SP5 II, Leica Microsystems, Wetzlar, Germany) at 400×magnifications.

\section{Cell culture and miRNA transfection}

HEK293T cells were purchased from the Cell Bank of Chinese Academy of Medical Science (Shanghai, China) and maintained in RPMI-1640 medium supplemented with 10\% FCS, $100 \mathrm{U} / \mathrm{ml}$ penicillin and 100 $\mathrm{U} / \mathrm{ml}$ streptomycin (Invitrogen) in a humidified incubator with $5 \% \mathrm{CO}_{2}$ at $37^{\circ} \mathrm{C}$. For the HEK293T cells transfection, miR-133b mimics or miR-133b inhibitor or their corresponding negative controls (Rio 


\section{Cellular Physiology Cell Physiol Biochem 2017;42:901-912 \begin{tabular}{l|l} 
and Biochemistry Published onlıne: June 26, 2017 & $\begin{array}{l}\text { (c) } 2017 \text { The Author(s). Published by S. Karger AG, Basel } \\
\text { www.karger.com/cpb }\end{array}$
\end{tabular} \\ Xiao et al.: Inhibitory Effects of miR-133b on Allergic Inflammation}

Biotechnology, Guangzhou, China) were diluted with OptiMEM I medium (Invitrogen) and transfected into cells with Lipofectamin 2000 (Invitrogen) according to manufacturer's instructions.

\section{Luciferase experiment}

Luciferase reporter assay was performed to predict the direct binding of miR-133b to the target gene Nlrp3. A wild-type murine Nlrp3 mRNA 3'UTR segment (Genbank accession No. NM_145827) was amplified and cloned into the pMIR-ReportTM vector (RiboBio Co., Guangzhou, China). Mutation in miR-133b bindingsite of Nlrp3 was generated by substitution of $6 \mathrm{bp}$ from seed region of miR-133b. HEK293T cells were cotransfected with either miR-133b mimics or miR-133b inhibitor or negative controls, and 3'UTR of Nlrp3 (with either wild-type or mutant miR-133b binding sites) using Lipofectamine 2000 for $48 \mathrm{~h}$ followed by the Dual-Luciferase Reporter Assay System (Promega, Madison, WI, USA).

\section{Western blotting}

HEK293T cells were lysed in RIPA buffer (Beyotime, Jiangsu, China) containing protease inhibitor (Merck, Darmstadt, Germany), and the proteins of the lysates were quantified using an Enhanced protein assay kit (Pierce, Rockford, IL, USA). Equal amounts of protein were separated by $10 \%$ SDS-PAGE, transferred to polyvinylidene difluoride membrane (PVDF, Millipore, Billerica, MA, USA), and then blocked with 5\% non-fat milk in TBST (20 mM Tris- $\mathrm{HCl}, 0.15 \mathrm{M} \mathrm{NaCl}, 0.05 \%$ Tween-20, pH 7.5) for $1 \mathrm{~h}$ at room temperature. The membranes were probed with primary antibodies against Nrlp3 (1:500 dilution, Santa Cruz) or GAPDH (1:1000 dilution, Beyotime) overnight at $4^{\circ} \mathrm{C}$. After washing with TBST for 3 times, the membranes were incubated with secondary antibody (goat anti-rabbit IgG conjugated with the horseradish peroxidase, 1:4000 dilution, Cell Signaling Technology) for $1 \mathrm{~h}$ at room temperature. Specific bands were visualized using the ECL chemiluminescence reaction kit (Cell Signaling Technology).

\section{Statistical analysis}

All data were presented as mean \pm SEM. Statistical significance was assessed with one-way analysis of variance (ANOVA) followed by Student's t-test for comparisons between the groups using SPSS Statistics 19.0 software (Chicago, IL, USA). Differences with P-values less than 0.05 were considered to be significant.

\section{Results}

Decreased expression of miR-133b in the nasal mucosa of AR mice

Previous study showed that miR-133b expression was reduced in plasma of patients with AR [19]. To further unveil the role of miR-133b in AR, we overexpressed miR-133b expression in the nasal mucosa of AR mice by intranasal administration of miR-133b agomir. As shown in Fig. 2, miR-133b expression was significantly downregulated in AR mice, suggesting miR-133b might be a potential negative regulator of the pro-allergic properties of AR. In addition, miR-133b expression was markedly restored in miR-133b agomir-treated AR mice, indicating successful gene transduction in nasal mucosa of these mice.

Fig. 2. Expression of miR-133b in nasal mucosa of AR mice following intranasal administration with miR-133b agomir. AR mice were induced as described in methods section, miR-133b agomir or mismatched agomir (MA) was then intranasally administrated to mice after OVA challenge for 7 days. The expression of miR-133b in nasal mucosa was determined by quantitative real-time PCR. The results were expressed as mean \pm SEM. ${ }^{* *} \mathrm{P}<0.01$ vs. control; $\# \# \mathrm{P}<0.01$ vs. $\mathrm{AR}, \mathrm{n}=8$ in each group.

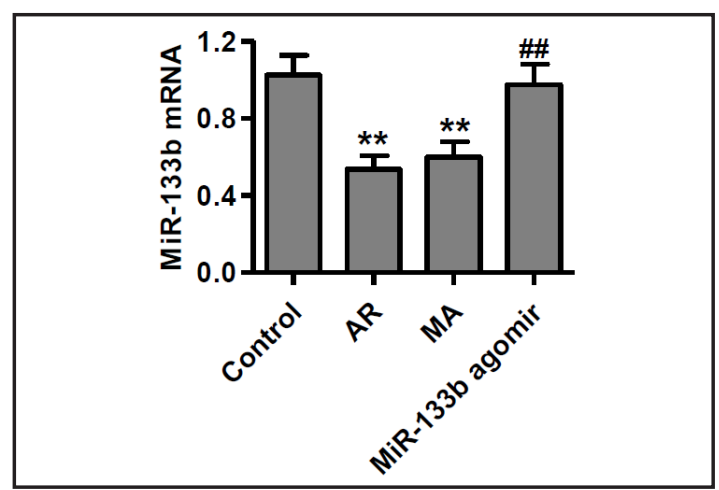




\section{Cellular Physiology and Biochemistry

Fig. 3. Effects of miR-133b on OVA-specific IgE and allergic symptoms in AR mice. (A) The concentration of OVA-specific IgE in serum were measured using ELISA. (B) The frequencies of rubbing and sneezing were recorded within 30 min of the final OVA challenge on day 34 , and symptom scores were calculated as described in methods section. The results were expressed as mean \pm SEM. ${ }^{* *} \mathrm{P}<0.01$ vs. control; \#\#P<0.01 vs. AR, $n=15$ in each group.
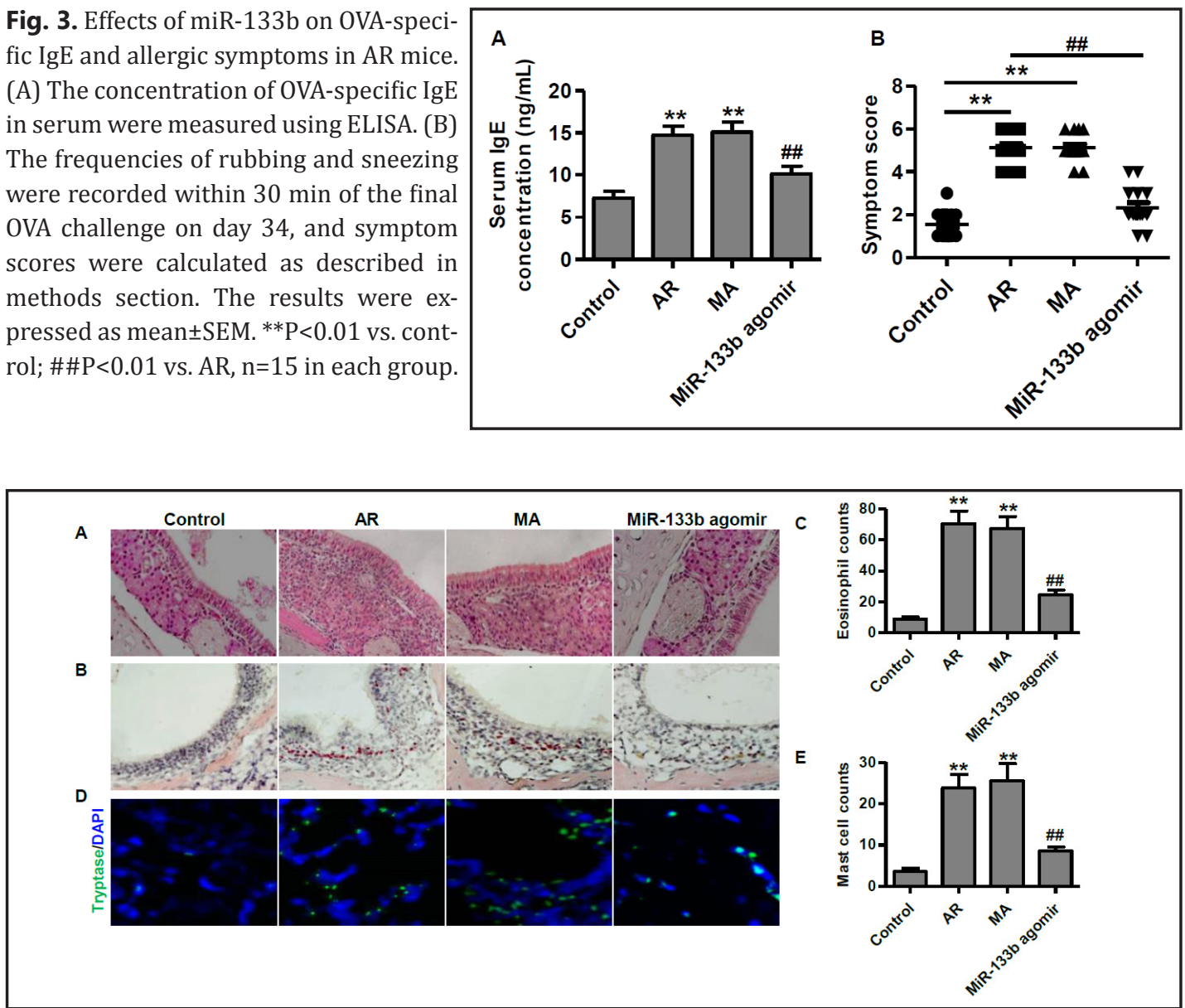

Fig. 4. MiR-133b inhibits immune cells infiltration in nasal mucosa of AR mice. (A) The pathological changes in nasal mucosa from each group were analyzed using H\&E staining. Representative images were shown (×200 magnifications). (B) Eosinophil infiltration in nasal mucosa was determined by Sirius red staining ( $\times 200$ magnifications). (C) The number of eosinophils was quantified for each group. ${ }^{* *} \mathrm{P}<0.01$ vs. control; $\# \# \mathrm{P}<0.01$ vs. AR, $n=6$ in each group. (D) The expression of tryptase (red) in mast cells was analyzed using a laser scanning confocal microscopy ( $\times 400$ magnifications). Cell nuclei were stained with DAPI (blue). (E) The number of mast cells was quantified for each group. ${ }^{* *} \mathrm{P}<0.01$ vs. control; $\# \# \mathrm{P}<0.01 \mathrm{vs}$. AR, $\mathrm{n}=4$ in each group.

Overexpression of miR-133b inhibits OVA-specific IgE and allergic symptoms in AR mice

To investigate the effects of miR-133b on allergic response in AR mice, we measured OVA-specific IgE in serum. The concentration of OVA-specific IgE was significantly increased in AR mice compared to the normal mice. However, intranasal administration of miR-133b agomir inhibited OVA-specific IgE concentration (Fig. 3A). Furthermore, the nasal allergic symptoms, including the number of nasal rubbing and sneezing, were counted after the last sensitization on day 34. The symptom score increased markedly in AR mice treated with or without mismatched agomir, but only increased slightly in miR-133b agomir-treated AR mice as compared with normal mice (Fig. 3B).

Inhibitory effect of miR-133b on the infiltration of inflammatory cells into the nasal mucosa tissues

We next explored the effects of miR-133b on the pathological alterations in nasal mucosa of AR mice. OVA sensitization induced apparent pathological changes, including hyperemia, edema, necrosis, and aberrant structure in nasal mucosa epithelium. All these pathological 


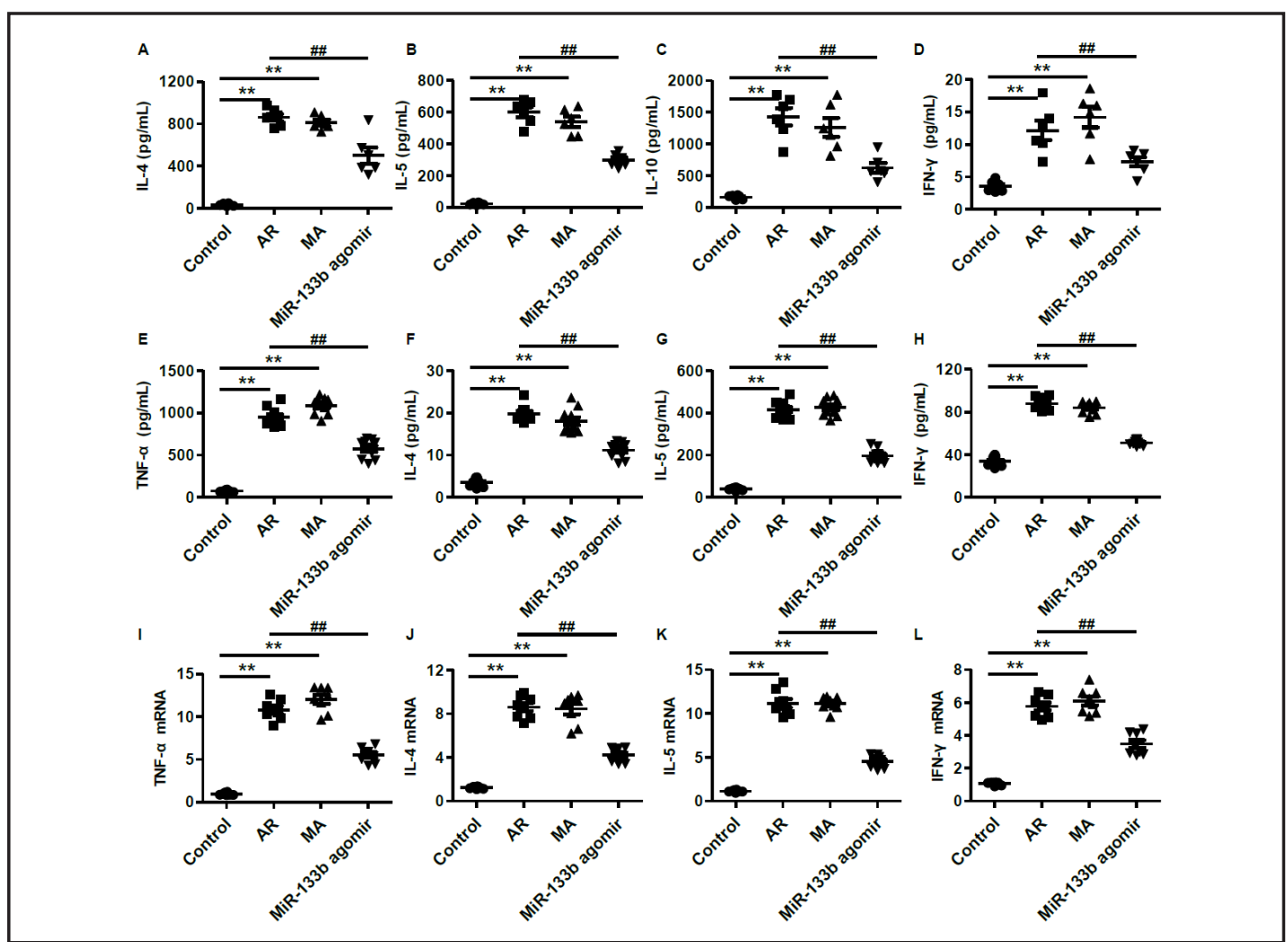

Fig. 5. Upregulation of MiR-133b reduces the cytokines levels in AR mice. (A-D) Cervical lymph nodes cell suspensions were retreated with OVA $(10 \mu \mathrm{g} / \mathrm{mL})$ in vitro for 4 days and analyzed for IL-4 (A), IL-5 (B), IL-10 (C) and IFN- $\gamma$ (D) levels using ELISA assay. (E-H) The levels of TNF- $\alpha$ (E), IL-4 (F), IL-5 (G) and IFN- $\gamma(\mathrm{H})$ in serum from each group were examined using ELISA. (I-L) The mRNA expressions of the above genes in nasal mucosa were determined by quantitative real-time PCR. Data were presented as mean $\pm \mathrm{SEM}$. ${ }^{* *} \mathrm{P}<0.01$ vs. control; \#\#P<0.01 vs. AR, $\mathrm{n}=6$-10 in each group.

alterations were ameliorated after miR-133b agomir administration (Fig. 4A). To further determine the effect of miR-133b on the influx of inflammatory cells into the nasal mucosa of AR, eosinophils and basophilic mast cells were stained. Sirius red staining showed that the number of eosinophils in nasal mucosa of AR mice was significantly increased as compared with normal mice. However, intranasal administration of miR-133b agomir markedly reduced the number of eosinophils (Fig. 4B and C). Moreover, the significant reduction in the number of mast cells was also observed in the nasal mucosa when AR mice were treated with miR-133b agomir (Fig. 4D and E). These data imply that miR-133b could alleviate the pathology and immune cells infiltration in the nasal mucosa of AR mice.

\section{MiR-133b upregulation reduces the levels of cytokines in AR mice}

Although the infiltration of eosinophils and basophilic mast cells indirectly suggest that intranasal application of OVA antigen can induce Th2 lymphocyte response, these immune cells in nasal mucosa can also be induced through the activation of innate lymphoid cells that producing IL-5 and/or IL-13 [21]. Thus, we investigated whether upregulation of miR-133b decreases Th2 immunity to OVA in vitro. T-lymphocytes obtained from the cervical lymph nodes of AR mice produced higher Th2 cytokines (IL-4, IL-5 and IL-10) but also higher Th1 cytokines (IFN- $\gamma$ ). These cytokines levels in miR-133b agomir-treated AR mice were significantly decreased compared with the AR mice alone (Fig. 5A-D). To further confirm the effect of miR-133b on proinflammatory cytokines, we measured the levels of TNF- $\alpha$, IL4 , IL-5 and IFN- $\gamma$ in serum. Not surprisingly, the levels of TNF- $\alpha$, IL-4, IL-5 and IFN- $\gamma$ were 


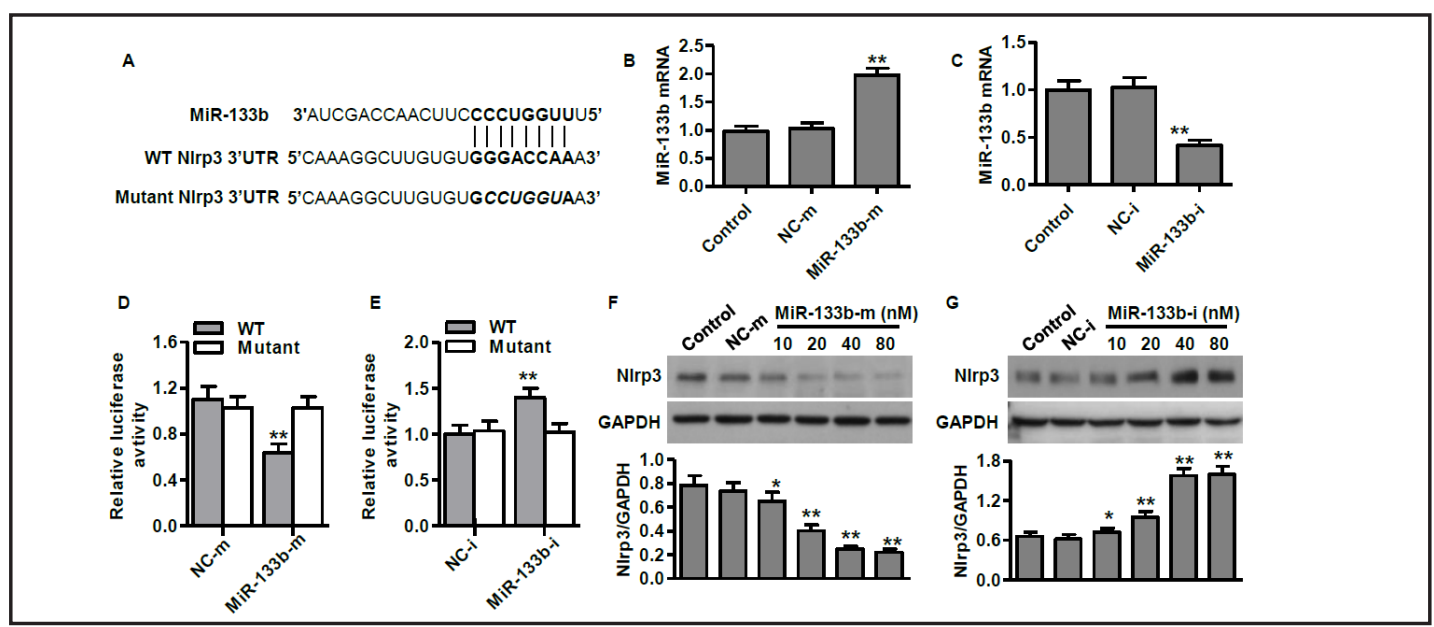

Fig. 6. MiR-133b negatively regulates Nlrp3 expression. (A) A predicted miR-133b binding site in the 3'UTR of Nlrp3. The complementary sequences were shown. (B and C) HEK293T cells were transected with miR133b mimics (miR-133b-i) (B) or miR-133b inhibitor (miR-133b-i) (C) or their corresponding negative control. The expression of miR-133b was determined by quantitative real-time PCR. ${ }^{* *} \mathrm{P}<0.01$ vs. control, $\mathrm{n}=6$. (D and E) The cells were co-transfected with either miR-133b mimics (miR-133b-m) (B) or miR-133b inhibitor (miR-133b-i) (C) or their corresponding negative control (mimics negative control (NC-i) or inhibitor negative control (NC-i)), and either wild-type (WT) or mutant Nlrp3 3'UTR. The luciferase assay was performed and verified that Nlrp3 as target gene of miR-133b. ${ }^{* *} \mathrm{P}<0.01$ vs. mimics negative control or inhibitor negative control, $n=8$. (F) Transfection of miR-133b mimics markedly decreased Nlrp3 protein expression. (G) Inhibition of miR-133b upregulated Nlrp3 expression. ${ }^{*} \mathrm{P}<0.05,{ }^{*} \mathrm{P}<0.01$ vs. control, $\mathrm{n}=6$.

remarkably upregulated in AR mice as compared with normal mice. However, miR-133b agomir administration was associated with reduced levels of these cytokines (Fig. 5E-H). In the line with the above results, the mRNA expressions of TNF- $\alpha$, IL-4, IL- 5 and IFN- $\gamma$ were also increased in nasal mucosa tissues of AR mice, which were markedly attenuated by miR133b agomir administration (Fig. 5I-L).

Verification of Nlrp3 as target gene of miR-133b

To understand the mechanisms by which miR-133b inhibited allergic responses and symptoms in AR mice, we used the miRNA target gene prediction website (http://www. microrna.org/) to predict the potential target gene of miR-133b. Notably, it was predicted that miR-133b might directly target Nlrp3, a gene known to be involved in the regulation of inflammation [10]. As displayed in Fig. 6A, an 8-bp fragment of the 3'UTR of Nlrp3 gene is complementary to the miR-133b seed sequence. To verify this prediction, we inserted the 3'UTR sequence of Nlrp3 into the luciferase reporter construct to determine the luciferase activity of Nlrp3 3'UTR. Successful overexpression or knockdown of miR-133b in HEK293T cells was evidenced by quantitative real-time PCR (Fig. 6B and C). The luciferase assay showed that upregulation of miR-133b by transfection of miR-133b mimics significantly decreased the luciferase activity in cells transfected with wild-type Nlrp3 3'UTR reporter, but not in cells transfected with the mutant one (Fig. 6D). Additionally, inhibition of miR133b using miR-133b inhibitor markedly increased the luciferase activity of Nlrp3 3'UTR, whereas this enhanced effect was completely abolished in the cells transfected with mutated Nlrp3 3'UTR (Fig. 6E). We next determined the effects of miR-133b on endogenous Nlrp3 protein expression. Consistently, overexpression of miR-133b remarkably reduced Nlrp3 protein expression, whereas miR-133b inhibition upregulated Nlrp3 expression (Fig. 6F and $\mathrm{G})$. These results suggest that upregulation of miR-133b may attenuate the inflammatory responses in AR mice nasal mucosa by negatively regulating Nlrp3 expression. 
Fig. 7. MiR-133b overexpression suppresses Nlrp3 inflammasome activation. (A-E) The expressions of Nlrp3 (A), Caspae-1 (B), ASC (C), IL-18 (D) and IL$1 \beta(E)$ in nasal mucosa from each group were determined by immunohistochemistry. (F-J) Quantitative real-time PCR was used to analyze the above genes expressions in nasal mucosa. Data were expressed as mean \pm SEM. ${ }^{* *} \mathrm{P}<0.01$ vs. control; \#\#P<0.01 vs. $\mathrm{AR}, \mathrm{n}=8$ in each group.

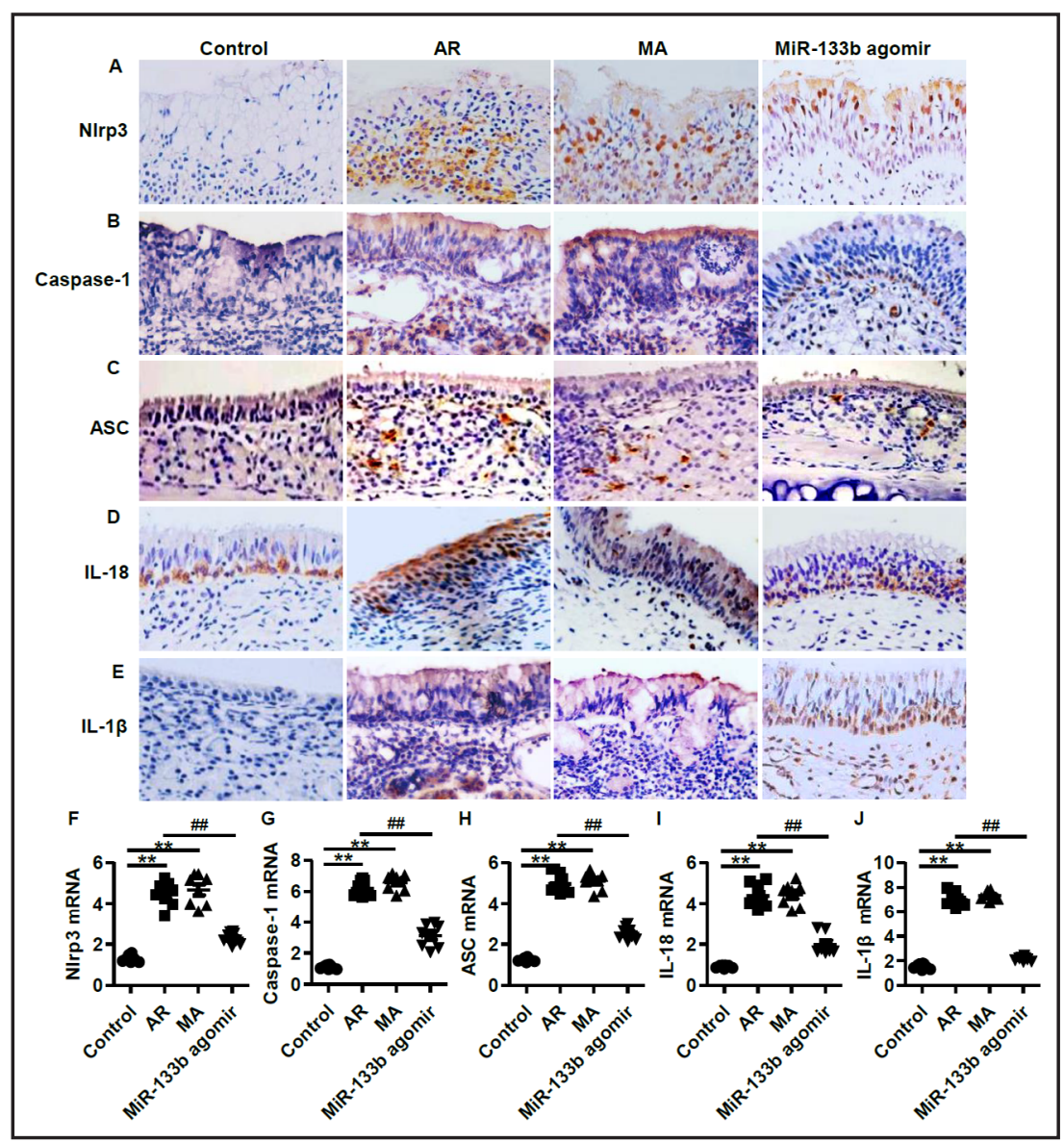

MiR-133b suppresses Nlrp3 inflammasome activation in nasal mucosa of AR mice

Given the important role of miR-133b in regulating Nlrp3 expression, we further investigated whether miR-133b inhibited Nlrp3 inflammasome activation and allergic inflammation in AR. The expressions of Nlrp3 inflammasome complex were examined in nasal mucosa. Immunohistochemistry showed that Nlrp3 expression was significantly higher in AR mice than in normal mice. Intranasal administration of miR-133b agomir remarkably attenuated OVA sensitization-induced the increase of Nlrp3 expression (Fig. 7A). As an indication of Nlrp3 inflammasome activation, we also observed increased expressions of Caspase-1, ASC, IL-18 and IL-1 $\beta$ in nasal mucosa of AR mice. However, these protein expressions were significantly downregulated in AR mice treated with miR-133b agomir (Fig. 7B-E). Consistent with the results of immunohistochemistry, quantitative real-time PCR showed that the mRNA expression of Nlrp3, Caspase-1, ASC, IL-18 and IL-1 $\beta$ in AR mice treated with miR-133b agomir was significantly decreased as compared with AR mice alone (Fig. 7F-J).

\section{Discussion}

In the last two decades, miRs are emerging as critical regulatory molecules that might be involved in the pathogenesis of various diseases $[19,22]$. They have been shown to degrade their cognate target gene expression at the post-transcriptional level by binding the 3'UTRs of their target mRNA [15]. Since miRs are more stable than many other mRNA moieties and even some proteins, many researchers have indicated that this stability could be exploited to clinical modulate gene expression in several diseases, including AR [22, 23]. For example, miR-106b negatively regulated the pro-allergic properties of bone marrow-derived dendritic 
cells and subsequent Th2 polarisation upon OVA stimulation. Thus, miR-106b may represent a promising therapeutic target for allergic inflammation [14]. Furthermore, intranasal administration of miR-135a lentivirus inhibited allergen-induced inflammation by targeting GATA-3 [15]. In addition, miR-126 was also found to be upregulated in the airway of asthma mice, and inhibition of miR-126 attenuated allergic inflammation [16]. In the present study, we demonstrate for the first time that miR-133b targets Nlrp3 expression and suppresses Nlrp3 inflammasome activation, leading to ameliorate allergic inflammation in AR model mice. These results provide a direct link between miR-133b and Nlrp3 inflammasomeinduced allergic inflammation in the pathogenesis of AR.

$\mathrm{MiR}-133 \mathrm{~b}$ has been regarded as an important predictor to diagnose and characterize several diseases [17-19]. Recently, a study utilizing bioinformatics data indicated that six circulating miRs, including miR-133b, were most predictive of allergic status, and miR$133 \mathrm{~b}$ showed decreased plasma level in AR patients [19]. Despite the role of miR-133b in inflammation has been increasingly recognized by basic and clinical researchers, reports of miR-133b in relation to allergic inflammation and rhinitis are very limited. Consistent with the previous study [19], we also found that miR-133b expression was decreased in nasal mucosa of AR model mice, further suggesting that miR-133b may negatively regulate the pro-allergic properties of AR. To avoid possible systemic complications that could be caused by tail vein injection, we intranasally administrated with miR-133b agomir in AR mice and investigated the directed effects of miR-133b on nasal mucosa. As expected, miR-133b expression was markedly restored after administration with miR-133b agomir. Moreover, the serum concentration of OVA-specific IgE and symptom of rubbing and sneezing were much lower in these mice than in AR model mice., indicating that intranasal administration of miR-133b agomir may in fact ameliorate allergic symptoms from AR.

Furthermore, AR is an inflammatory disorder of the nasal mucosa in the upper airways with infiltration of inflammatory cells such as basophilic mast cells and eosinophils [15, 24]. Early-phase reactions in allergic inflammation are regulated by basophilic mast cells, whereas eosinophils play a key role in the late-phase reactions of AR [13, 25]. The influx of these inflammatory cells into nasal mucosa, in turn, results in production of leukotrienes and histamine, as well as proinflammatory cytokines, which is characterized by nasal hyposmia, hyperreactivity and blockage [25]. TNF $\alpha$, an important proinflammatory cytokines produced by mast cells and eosinophils, is critical for the pathogenesis of AR $[5,26]$. In previous studies, the level of TNF $\alpha$ has been found to be effectively inhibited by intranasal administration with steroids [27]. More importantly, allergic inflammatory diseases are predominantly control by Th2-mediated immunological responses [4, 8]. Depletion of Th2 cells has been demonstrated to inhibit allergic inflammation typical of AR [28, 29]. Th2 cytokines, such as IL4 and IL5, are produced by mast cells, T cells and macrophages [1]. They play an important role in regulating the differentiation of $\mathrm{T}$ cells from the Th2 type and the IgE isotype switching in B cells $[1,30]$. However, recent studies showed that the level of the Th1 cytokine, IFN- $\gamma$, was increased in AR, which could be also inhibited by steroids treatment [4, 6 , 31]. Upregulation of IFN- $\gamma$ levels may act as a "supporter" for Th2 cells cytokines activity in allergic response rather than playing an inhibitory role in AR [24, 31]. Here, we found that intranasal administration with miR-133b agomir markedly inhibited eosinophils and mast cells infiltration in nasal mucosa of AR mice. Moreover, miR-133b upregulation reduced the levels of TNF $\alpha$, IL-4, IL-5, IL-10 and IFN- $\gamma$ in AR mice. The results imply that the inhibition of Th1 and Th2 cytokines may underlie the anti-inflammatory effect of miR-133b in AR.

Increasing evidences demonstrated that Caspase- 1 and IL-1 $\beta$ expressions were upregulated in the nasal mucosa tissues of $\operatorname{AR}$ mice $[13,32]$. Inhibition of allergic inflammatory reactions was found in Caspase- 1 deficient AR mice [12]. In addition, activation of Caspase-1 or IL- $1 \beta$ accelerates inflammatory reactions by causing the recruitment of eosinophils into nasal mucosa tissue [20]. It is worthy to note that Caspase- 1 and IL-1 $\beta$ are two key components of Nlrp3 inflammasome $[9,10,32]$. Upon inflammatory stimuli, the Nlrp3 inflammasome induces Caspase- 1 activation, resulting in mature IL-18 and IL-1 $\beta$ generation from their respective precursors, pro- IL-18 and IL-1 $\beta[11,33]$. Previous study

\section{KARGER}




\section{Cellular Physiology Cell Physiol Biochem 2017;42:901-912

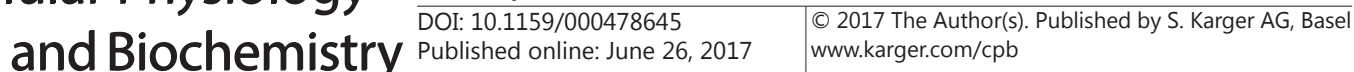

indicated that the inflammatory response in a rat AR model was associated with Nlrp3 inflammasome activation [11]. In this study, our data revealed that miR-133b determined Nlrp3 expression by directly binding to the 3'UTR of Nlrp3. In nasal mucosa of AR mice, the expression of Nlrp3 and its downstream cytokines Caspase-1, ASC, IL-18 and IL-1 $\beta$ were all upregulated. However, intranasal administration with miR-133b agomir inhibited the above gene expressions, suggesting that miR-133b attenuates allergic inflammation by inhibiting Nlrp3 inflammasome activation in AR mice.

In conclusion, our present study demonstrate that upregulation of miR-133b effectively ameliorates the pathology of AR through inhibition of inflammatory responses, which includes reduced serum OVA-specific IgE concentration, eosinophils and mast cells infiltration and proinflammatory cytokines levels. Notably, we unravel that miR-133b directly targets Nlrp expression and subsequently suppresses Nlrp3 inflammasome activation. These findings suggest that miR-133b alleviates allergic inflammation of AR apparently by inhibition of Nlrp3 inflammasome activation and upregulation of miR-133b in nasal mucosa may be a potential therapeutic approach for the treatment of AR.

\section{Disclosure Statement}

The authors have declared that no competing interests exist.

\section{References}

1 Settipane RA, Schwindt C: Chapter 15: Allergic rhinitis. Am J Rhinol Allergy 2013;27:S52-55.

-2 Rosati MG, Peters AT: Relationships among allergic rhinitis, asthma, and chronic rhinosinusitis. Am J Rhinol Allergy 2016;30:44-47.

-3 Steelant B, Farre R, Wawrzyniak P, Belmans J, Dekimpe E, Vanheel H, Van Gerven L, Kortekaas Krohn I, Bullens DM, Ceuppens JL, Akdis CA, Boeckxstaens G, Seys SF, Hellings PW: Impaired barrier function in patients with house dust mite-induced allergic rhinitis is accompanied by decreased occludin and zonula occludens-1 expression. J Allergy Clin Immunol 2016;137:1043-1053 e1041-1045.

4 Bae JS, Kim JH, Kim EH, Mo JH: The Role of IL-17 in a Lipopolysaccharide-Induced Rhinitis Model. Allergy Asthma Immunol Res 2017;9:169-176.

-5 Mo JH, Kang EK, Quan SH, Rhee CS, Lee CH, Kim DY: Anti-tumor necrosis factor-alpha treatment reduces allergic responses in an allergic rhinitis mouse model. Allergy 2011;66:279-286.

6 Wang H, Barrenas F, Bruhn S, Mobini R, Benson M: Increased IFN-gamma activity in seasonal allergic rhinitis is decreased by corticosteroid treatment. J Allergy Clin Immunol 2009;124:1360-1362. Romagnani S: The role of lymphocytes in allergic disease. J Allergy Clin Immunol 2000;105:399-408. Bachert C, Zhang L, Gevaert P: Current and future treatment options for adult chronic rhinosinusitis: Focus on nasal polyposis. J Allergy Clin Immunol 2015;136:1431-1440; quiz 1441.

9 Schroder K, Tschopp J: The inflammasomes. Cell 2010;140:821-832.

10 Huang Z, Zhuang X, Xie C, Hu X, Dong X, Guo Y, Li S, Liao X: Exogenous Hydrogen Sulfide Attenuates High Glucose-Induced Cardiotoxicity by Inhibiting NLRP3 Inflammasome Activation by Suppressing TLR4/NFkappaB Pathway in H9c2 Cells. Cell Physiol Biochem 2016;40:1578-1590.

11 Wan H, Su H, Wu Y, Zhao Y, Zhou M: [Expression and significance of NLRP3 inflammasome and its downstream factors IL-1beta/IL-18 in rat model of allergic rhinitis]. Zhonghua Er Bi Yan Hou Tou Jing Wai Ke Za Zhi 2015;50:145-150.

12 Martin RA, Ather JL, Lundblad LK, Suratt BT, Boyson JE, Budd RC, Alcorn JF, Flavell RA, Eisenbarth SC, Poynter ME: Interleukin-1 receptor and caspase-1 are required for the Th17 response in nitrogen dioxidepromoted allergic airway disease. Am J Respir Cell Mol Biol 2013;48:655-664.

13 Kleinjan A, van Nimwegen M, Leman K, Hoogsteden HC, Lambrecht BN: Topical treatment targeting sphingosine-1-phosphate and sphingosine lyase abrogates experimental allergic rhinitis in a murine model. Allergy 2013;68:204-212. 


\section{Cellular Physiology Cell Physiol Biochem 2017;42:901-912 \begin{tabular}{l|l} 
DOI: 10.1159/000478645 & Ond Biochemistry 2017 The Author(s). Published by S. Karger AG, Basel \\
wwww.karger.com/cpb
\end{tabular} and Biochemistry}

14 Tang H, Jiang H, Zheng J, Li J, Wei Y, Xu G, Li H: MicroRNA-106b regulates pro-allergic properties of dendritic cells and Th2 polarisation by targeting early growth response-2 in vitro. Int Immunopharmacol 2015;28:866-874.

15 Deng YQ, Yang YQ Wang SB, Li F, Liu MZ, Hua QQ Tao ZZ: Intranasal Administration of Lentiviral miR-135a Regulates Mast Cell and Allergen-Induced Inflammation by Targeting GATA-3. PLoS One 2015;10:e0139322.

-16 Mattes J, Collison A, Plank M, Phipps S, Foster PS: Antagonism of microRNA-126 suppresses the effector function of TH2 cells and the development of allergic airways disease. Proc Natl Acad Sci U S A 2009;106:18704-18709.

-17 Xu Y, Zhang X, Pu S, Wu J, Lv Y, Du D: Circulating microRNA expression profile: a novel potential predictor for chronic nervous lesions. Acta Biochim Biophys Sin (Shanghai) 2014;46:942-949.

-18 Georgantas RW, Streicher K, Greenberg SA, Greenlees LM, Zhu W, Brohawn PZ, Higgs BW, Czapiga M, Morehouse CA, Amato A, Richman L, Jallal B, Yao Y, Ranade K: Inhibition of myogenic microRNAs 1, 133, and 206 by inflammatory cytokines links inflammation and muscle degeneration in adult inflammatory myopathies. Arthritis Rheumatol 2014;66:1022-1033.

19 Panganiban RP, Wang Y, Howrylak J, Chinchilli VM, Craig TJ, August A, Ishmael FT: Circulating microRNAs as biomarkers in patients with allergic rhinitis and asthma. J Allergy Clin Immunol 2016;137:1423-1432.

20 Galli SJ, Tsai M: IgE and mast cells in allergic disease. Nat Med 2012;18:693-704.

-21 Klein Wolterink RG, Kleinjan A, van Nimwegen M, Bergen I, de Bruijn M, Levani Y, Hendriks RW: Pulmonary innate lymphoid cells are major producers of IL-5 and IL-13 in murine models of allergic asthma. Eur J Immunol 2012;42:1106-1116.

22 Zhang XH, Zhang YN, Liu Z: MicroRNA in chronic rhinosinusitis and allergic rhinitis. Curr Allergy Asthma Rep 2014;14:415.

-23 Teng Y, Zhang R, Yu H, Wang H, Hong Z, Zhuang W, Huang Y: Altered MicroRNA Expression Profiles in Activated Mast Cells Following IgE-FcepsilonRI Cross-Linking with Antigen. Cell Physiol Biochem 2015;35:2098-2110.

-24 Benson M, Strannegard IL, Strannegard O, Wennergren G: Topical steroid treatment of allergic rhinitis decreases nasal fluid TH2 cytokines, eosinophils, eosinophil cationic protein, and IgE but has no significant effect on IFN-gamma, IL-1beta, TNF-alpha, or neutrophils. J Allergy Clin Immunol 2000;106:307-312.

25 Juliusson S, Holmberg K, Karlsson G, Enerback L, Pipkorn U: Mast cells and mediators in the nasal mucosa after allergen challenge. Effects of four weeks' treatment with topical glucocorticoid. Clin Exp Allergy 1993;23:591-599.

26 Jung HW, Jung JK, Park YK: Comparison of the efficacy of KOB03, ketotifen, and montelukast in an experimental mouse model of allergic rhinitis. Int Immunopharmacol 2013;16:254-260.

27 Meltzer EO: The role of nasal corticosteroids in the treatment of rhinitis. Immunol Allergy Clin North Am 2011;31:545-560.

28 Kobayashi N, Terada N, Hamano N, Numata T, Konno A: Transepithelial migration of activated eosinophils induces a decrease of E-cadherin expression in cultured human nasal epithelial cells. Clin Exp Allergy 2000;30:807-817.

-29 Yu HQ, Liu ZG, Yu KY, Xu ZQ, Qiu J: [Immunotherapy with recombinant house dust mite group 2 allergen vaccine inhibits allergic airway inflammation in mice]. Zhongguo Ji Sheng Chong Xue Yu Ji Sheng Chong Bing Za Zhi 2006;24:414-419.

-30 Ying S, Durham SR, Corrigan CJ, Hamid Q, Kay AB: Phenotype of cells expressing mRNA for TH2-type (interleukin 4 and interleukin 5) and TH1-type (interleukin 2 and interferon gamma) cytokines in bronchoalveolar lavage and bronchial biopsies from atopic asthmatic and normal control subjects. Am J Respir Cell Mol Biol 1995;12:477-487.

-31 Gunel C, Demirci B, Eryilmaz A, Yilmaz M, Meteoglu I, Omurlu IK, Basal Y: Inhibitory Effect of Pycnogenol(R) on Airway Inflammation in Ovalbumin-Induced Allergic Rhinitis. Balkan Med J 2016;33:620-626.

-32 Kim HY, Nam SY, Hwang SY, Kim HM, Jeong HJ: Atractylone, an active constituent of KMP6, attenuates allergic inflammation on allergic rhinitis in vitro and in vivo models. Mol Immunol 2016;78:121-132.

-33 Liu W, Zhang X, Zhao M, Zhang X, Chi J, Liu Y, Lin F, Fu Y, Ma D, Yin X: Activation in M1 but not M2 Macrophages Contributes to Cardiac Remodeling after Myocardial Infarction in Rats: a Critical Role of the Calcium Sensing Receptor/NRLP3 Inflammasome. Cell Physiol Biochem 2015;35:2483-2500. 